\title{
New parametric generalized exponential fuzzy divergence measure
}

\author{
Vijay Prakash Tomar and Anshu Ohlan*
}

\author{
* Correspondence: \\ anshu.gahlawat@yahoo.com \\ Department of Mathematics, \\ Deenbandhu Chhotu Ram \\ University of Science and \\ Technology, Murthal 131039, \\ Haryana, India
}

\begin{abstract}
The present paper introduces a parametric generalized exponential measure of fuzzy divergence of order a with the proof of its validity. A particular case of proposed fuzzy divergence measure is studied. Some properties of the new divergence measure between different fuzzy sets are proved. We establish a relation between exponential fuzzy entropy of order a and our fuzzy divergence measure. Further, a numerical example is given for the comparative study of the new divergence measure with some of existing measures. Finally, application of the measure to strategic decision-making is discussed and a comparative study of the method of strategic decision-making with the existing methods is presented. It is noted that the new measure of fuzzy divergence and the method of strategic decision-making comprise greater simplicity, consistency and flexibility in applications due to the presence of the parameter.
\end{abstract}

Keywords: Fuzzy set; Fuzziness; Fuzzy entropy; Fuzzy directed divergence; Decision making

\section{Introduction}

Entropy is one of the key measures of information first used by Shannon [1]. Entropy as a measure of fuzziness was introduced by Zadeh [2]. Fuzzy entropy is an important concept for measuring fuzzy information. A measure of the fuzzy entropy of a fuzzy set is a measure of the fuzziness of the set. Kapur [3] argues that fuzzy entropy measures uncertainty due to fuzziness of information, while probabilistic entropy measures uncertainty due to the information being available in terms of a probability distribution only. The concept of fuzzy sets proposed by Zadeh [2] has proven useful in the context of pattern recognition, image processing, speech recognition, bioinformatics, fuzzy aircraft control, feature selection, decision-making, etc.

During the last six decades, entropy, as a very important notion for measuring fuzziness degree or uncertain information in fuzzy set theory, has received a great attention. Fuzzy sets gained a vital attention from researchers for their application in various fields. For example, De Luca and Termini [4] introduced the measure of fuzzy entropy corresponding to Shannon [1] entropy. Later on, Bhandari and Pal [5] defined the exponential fuzzy entropy corresponding to Pal and Pal [6] exponential entropy. Verma and Sharma [7] generalized the Pal and Pal [6] exponential fuzzy entropy of order $\alpha>0$. Kullback and Leibler [8] obtained the measure of directed divergence between two probability distributions. Bhandari and Pal [5] presented some axioms to describe the measure of directed 
divergence between fuzzy sets, which is proposed corresponding to Kullback and Leibler [8] measure of directed divergence.

Thereafter, many other researchers have studied the fuzzy divergence measures in different ways and provide their application in different areas. For example, Fan and Xie [9] introduced the divergence measure based on exponential operation and corresponding to the fuzzy exponential entropy of Bhandari and Pal [5] and studied its relation with divergence measure introduced in [5]. Ghosh et al. [10] gave application of fuzzy divergence measure in the area of automated leukocyte recognition. In 2002, Montes et al. [11] studied the special classes of divergence measures and used the link between fuzzy and probabilistic uncertainty. Prakash et al. [12] proposed two fuzzy divergence measures corresponding to Ferreri [13] probabilistic measure of directed divergence. Corresponding to Renyi [14] generalized measure of directed divergence and Sharma and Mittal [15] generalized measure of directed divergence, Bajaj and Hooda [16] proposed the measure of fuzzy-directed divergence respectively. Bhatia and Singh [17] proposed four fuzzy divergence measures; one of them was corresponding to Taneja [18] arithmetic-geometric divergence measure.

Inspired by the abovementioned work, we introduce a generalized methodology for measuring the degree of difference between two fuzzy sets. We present a new parametric generalized exponential measure of fuzzy divergence and study the essential properties of this measure in order to check its authenticity.

The paper consists of eight sections. The introductory section is followed by a discussion on some well-known concepts and the notation related to fuzzy set theory. In the 'New parametric generalized exponential measure of fuzzy divergence' section, we introduce a parametric generalized fuzzy exponential measure of divergence corresponding to generalized fuzzy entropy given by Verma and Sharma [7]. In the 'Properties of generalized exponential fuzzy divergence measure' section, we first provide some interesting properties of the proposed measure of fuzzy divergence and then a relation between generalized exponential fuzzy entropy and the proposed fuzzy divergence measure is established. In the 'A comparative study' section, the comparison of the proposed divergence with some of existing generalized measures of fuzzy divergence is presented with the help of table and graph. The application of the proposed parametric generalized exponential measure of fuzzy divergence to strategic decision-making is illustrated with the help of a numerical example in the 'Application of parametric generalized exponential measure of fuzzy divergence in strategic decision-making' section. The 'Application of fuzzy TOPSIS and MOORA methods for strategic decision-making: a comparative analysis' section presents the application of the proposed measure of fuzzy divergence in the existing methods of strategic decision-making and a comparative analysis between the proposed method of strategic decision-making and the existing methods. Finally, the paper is concluded in the 'Concluding remarks' section.

\section{Preliminaries}

This section is devoted to introduce some well-known concepts and the notations related to fuzzy divergence measures. First of all, we will focus on the theory of fuzzy sets. Then, we will recall the axiomatic definition of a divergence measure for fuzzy sets. 


\section{Fuzzy sets}

Fuzzy sets are used to solve a lot of real-world problems. Fuzziness, a feature of uncertainty, results from the lack of sharp difference of being or not being a member of the set, i.e., the boundaries of the set under consideration are not sharply defined.

A fuzzy set $A$ defined on a universe of discourse $X$ is given as Zadeh [2]:

$$
A=\left\{\left\langle x, \mu_{A}(x)\right\rangle / x \in X\right\}
$$

where $\mu_{A}: X \rightarrow[0,1]$ is the membership function of $A$. The membership value $\mu_{A}(x)$ describes the degree of the belongingness of $x \in X$ in $A$. When $\mu_{A}(x)$ is valued in $\{0,1\}$, it is the characteristic function of a crisp (i.e., non-fuzzy) set. Zadeh [19] gave some notions related to fuzzy sets, some of them which we shall need in our discussion are as follows:

(1) Compliment: $\bar{A}=$ Compliment of $A \Leftrightarrow \mu_{\bar{A}}(x)=1-\mu_{A}(x)$ for all $x \in X$.

(2) Union: $A \cup B=$ Union of $A$ and $B \Leftrightarrow \mu_{A} \cup B(x)=\max \left\{\mu_{A}(x), \mu_{B}(x)\right\}$ for all $x \in X$.

(3) Intersection: $A \cap B=$ Intersection of $A$ and $B \Leftrightarrow \mu_{A} \cap B(x)=\min \left\{\mu_{A}(x), \mu_{B}(x)\right\}$ for all $x \in X$

\section{Fuzzy divergence measure}

In fuzzy context, several measures have been proposed in order to measure the degree of difference between two fuzzy sets. A general study of the axiomatic definition of a divergence measure for fuzzy sets was presented in Bouchon-Meunier et al. [20] and as a particular case was widely studied in Montes et al. [11].

Bhandari et al. [21] introduced the measure of fuzzy directed divergence corresponding to Kullback and Leibler [8] measure of directed divergence, as

$$
I(A: B)=\sum_{i=1}^{n}\left[\mu_{A}\left(x_{i}\right) \log \frac{\mu_{A}\left(x_{i}\right)}{\mu_{B}\left(x_{i}\right)}+\left(1-\mu_{A}\left(x_{i}\right)\right) \log \frac{1-\mu_{A}\left(x_{i}\right)}{1-\mu_{B}\left(x_{i}\right)}\right]
$$

and also provides the essential conditions for a measure of divergence.

The measure of fuzzy divergence between two fuzzy sets gives the difference between two fuzzy sets, and this measure of distance/difference between two fuzzy sets is called the fuzzy divergence measure.

Fan and Xie [9] proposed the fuzzy information of discrimination of $A$ against $B$ corresponding to the exponential fuzzy entropy of Pal and Pal [6] and is defined by

$$
I(A, B)=\sum_{i=1}^{n}\left[1-\left(1-\mu_{A}\left(x_{i}\right)\right) e^{\mu_{A}\left(x_{i}\right)-\mu_{B}\left(x_{i}\right)}-\mu_{A}\left(x_{i}\right) e^{\left(\mu_{B}\left(x_{i}\right)-\mu_{A}\left(x_{i}\right)\right)}\right]
$$

Finally, we may mention some other generalized measures of fuzzy divergence with which we compare our study.

Kapur [3] presented a fuzzy divergence measure corresponding to Havada-Charvat [22] measure of directed divergence which is given by

$$
I_{\alpha}(A, B)=\frac{1}{\alpha-1} \sum_{i=1}^{n}\left[\mu_{A}^{\alpha} \mu_{B}^{1-\alpha}+\left(1-\mu_{A}\right)^{\alpha}\left(1-\mu_{B}\right)^{1-\alpha}-1\right], \alpha \neq 1, \alpha>0 .
$$

Prakash et al. [12] proposed a fuzzy divergence measure corresponding to Ferreri's [13] probabilistic measure of directed divergence given by 


$$
\begin{aligned}
& I_{a}(A: B)=\sum_{i=1}^{n}\left[\mu_{A}\left(x_{i}\right) \log \frac{\mu_{A}\left(x_{i}\right)}{\mu_{B}\left(x_{i}\right)}+\left(1-\mu_{A}\left(x_{i}\right)\right) \log \frac{1-\mu_{A}\left(x_{i}\right)}{1-\mu_{B}\left(x_{i}\right)}\right] \\
& -\frac{1}{a} \sum_{i=1}^{n}\left[\left(1+a \mu_{A}\left(x_{i}\right)\right) \log \frac{1+a \mu_{A}\left(x_{i}\right)}{1+a \mu_{B}\left(x_{i}\right)}+\left\{1+a\left(1-\mu_{A}\left(x_{i}\right)\right)\right\} \log \frac{1+a\left(1-\mu_{A}\left(x_{i}\right)\right)}{1+a\left(1-\mu_{B}\left(x_{i}\right)\right)}\right]
\end{aligned}
$$

Corresponding to Renyi [14] generalized measure of directed divergence Bajaj and Hooda [16] provided the generalized fuzzy divergence measure which is given by

$$
D_{\alpha}(A, B)=\frac{1}{\alpha-1} \sum_{i=1}^{n} \log \left[\mu_{A}^{\alpha} \mu_{B}^{1-\alpha}+\left(1-\mu_{A}\right)^{\alpha}\left(1-\mu_{B}\right)^{1-\alpha}\right], \alpha \neq 1, \alpha>0 .
$$

\section{New parametric generalized exponential measure of fuzzy divergence}

We now propose a new parametric generalized exponential measure of divergence between fuzzy sets $A$ and $B$ corresponding to generalized exponential fuzzy entropy of order $\alpha>0$ given by Verma and Sharma [7] as

$$
I_{E_{\alpha}}(A, B)=\sum_{i=1}^{n}\left[1-\left(1-\mu_{A}\left(x_{i}\right)\right) e^{\left(\left(1-\mu_{B}\left(x_{i}\right)\right)^{\alpha}-\left(1-\mu_{A}\left(x_{i}\right)\right)^{\alpha}\right)}-\mu_{A}\left(x_{i}\right) e^{\left(\mu_{B}^{\alpha}\left(x_{i}\right)-\mu_{A}^{\alpha}\left(x_{i}\right)\right)}\right]
$$

Theorem $1 I_{E_{\alpha}}(A, B)$ is a valid measure of fuzzy directed divergence.

Proof: It is clear from (6) that

(i) $I_{E_{\alpha}}(A, B) \geq 0$

(ii) $I_{E_{\alpha}}(A, B)=0$ if $\mu_{A}\left(x_{i}\right)=\mu_{B}\left(x_{i}\right)$

(iii) $I_{E_{\alpha}}(A, B) \neq I_{E_{\alpha}}(B, A)$

But $J_{E_{\alpha}}(A, B)=I_{E_{\alpha}}(A, B)+I_{E_{\alpha}}(B, A)$ is symmetric.

(iv) We now check the convexity of $I_{E_{\alpha}}(A, B)$ :

$$
\begin{aligned}
& \frac{\partial I_{E_{\alpha}}}{\partial \mu_{A}\left(x_{i}\right)}=\left(1-\alpha\left(1-\mu_{A}\left(x_{i}\right)\right)^{\alpha}\right) e^{\left(\left(1-\mu_{B}\left(x_{i}\right)\right)^{\alpha}-\left(1-\mu_{A}\left(x_{i}\right)\right)^{\alpha}\right)}+(\alpha-1) e^{\left(\mu_{B}^{\alpha}\left(x_{i}\right)-\mu_{A}^{\alpha}\left(x_{i}\right)\right)} \\
& \frac{\partial^{2} I_{E_{\alpha}}}{\partial \mu_{A}^{2}\left(x_{i}\right)}=\alpha(\alpha+1)\left[\left(1-\mu_{A}\left(x_{i}\right)\right)^{\alpha-1} e^{\left(1-\mu_{B}\left(x_{i}\right)\right)^{\alpha}-\left(1-\mu_{A}\left(x_{i}\right)\right)^{\alpha}}+\mu_{A}^{\alpha-1}\left(x_{i}\right) e^{\mu_{B}^{\alpha}\left(x_{i}\right)-\mu_{A}^{\alpha}\left(x_{i}\right)}\right] \\
& -\alpha^{2}\left[\left(1-\mu_{A}\left(x_{i}\right)\right)^{2 \alpha-1} e^{\left(1-\mu_{B}\left(x_{i}\right)\right)^{\alpha}-\left(1-\mu_{A}\left(x_{i}\right)\right)^{\alpha}}+\mu_{A}^{2 \alpha-1}\left(x_{i}\right) e^{\mu_{B}^{\alpha}\left(x_{i}\right)-\mu_{A}^{\alpha}\left(x_{i}\right)}\right]>0 \text { for } \alpha>0 .
\end{aligned}
$$

Similarly, $\frac{\partial^{2} I_{E_{\alpha}}}{\partial \mu_{B}^{2}\left(x_{i}\right)}>0$ for $\alpha>0$.

Thus, $I_{E_{\alpha}}(A, B)$ is a convex function of fuzzy sets $A$ and $B$ and therefore $I_{E_{\alpha}}(A, B)$ is a valid measure of fuzzy-directed divergence.

Particular case: For $\alpha=1, I_{E_{\alpha}}(A, B)$ reduces to $I(A, B)$ given in (2).

\section{Properties of generalized exponential fuzzy divergence measure}

The generalized exponential fuzzy divergence measure $I_{E_{\alpha}}(A, B)$ defined above has the following properties:

Theorem 2 (a) $I_{E_{\alpha}}(A \cup B, A)+I_{E_{\alpha}}(A \cap B, A)=I_{E_{\alpha}}(B, A)$

(b) $I_{E_{\alpha}}(A \cup B, C)+I_{E_{\alpha}}(A \cap B, C)=I_{E_{\alpha}}(A, C)+I_{E_{\alpha}}(B, C)$

(c) $I_{E_{\alpha}}(\overline{A \cup B}, \overline{A \cap B})=I_{E_{\alpha}}(\bar{A} \cap \bar{B}, \bar{A} \cup \bar{B}$,

Proof: 2(a) Let us consider the sets 


$$
\begin{aligned}
& X_{1}=\left\{x / x \in X, \mu_{A}\left(x_{i}\right) \geq \mu_{B}\left(x_{i}\right)\right\} \\
& \text { and } X_{2}=\left\{x / x \in X, \mu_{A}\left(x_{i}\right)<\mu_{B}\left(x_{i}\right)\right\}
\end{aligned}
$$

Using the notions explained above in the 'Preliminaries' section:

$$
\begin{aligned}
& \text { In set } X_{1}, A \cup B=\text { Union of } A \text { and } B \Leftrightarrow \mu_{A \cup B}(x)=\max \left\{\mu_{A}(x), \mu_{B}(x)\right\}=\mu_{A}(x) \\
& A \cap B=\text { Intersection of } A \text { and } B \Leftrightarrow \mu_{A \cap B}(x)=\min \left\{\mu_{A}(x), \mu_{B}(x)\right\}=\mu_{B}(x) \\
& \text { In set } X_{2}, A \cup B=\text { Union of } A \text { and } B \Leftrightarrow \mu_{A \cup B}(x)=\max \left\{\mu_{A}(x), \mu_{B}(x)\right\}=\mu_{B}(x) \\
& A \cap B=\text { Intersection of } A \text { and } B \Leftrightarrow \mu_{A \cap B}(x)=\min \left\{\mu_{A}(x), \mu_{B}(x)\right\}=\mu_{A}(x)
\end{aligned}
$$

We have

$$
\begin{aligned}
& I_{E_{\alpha}}(A \cup B, A)+I_{E_{\alpha}}(A \cap B, A)=\sum_{i=1}^{n}\left[1-\left(1-\mu_{A \cup B}\left(x_{i}\right)\right) e^{\left(\left(1-\mu_{A}\left(x_{i}\right)\right)^{\alpha}-\left(1-\mu_{A \cup B}\left(x_{i}\right)\right)^{\alpha}\right)}\right. \\
& \left.-\mu_{A \cup B}\left(x_{i}\right) e^{\left(\mu_{A}^{\alpha}\left(x_{i}\right)-\mu_{A \cup B}^{\alpha}\left(x_{i}\right)\right)}\right] \\
& +\sum_{i=1}^{n}\left[1-\left(1-\mu_{A \cap B}\left(x_{i}\right)\right) e^{\left(\left(1-\mu_{A}\left(x_{i}\right)\right)^{\alpha}-\left(1-\mu_{A \cap B}\left(x_{i}\right)\right)^{\alpha}\right)}\right. \\
& \left.-\mu_{A \cap B}\left(x_{i}\right) e^{\left(\mu_{A}^{\alpha}\left(x_{i}\right)-\mu_{A \cap B}^{\alpha}\left(x_{i}\right)\right)}\right] \\
& =\left\{\sum _ { X _ { 1 } } \left[1-\left(1-\mu_{A}\left(x_{i}\right)\right) e^{\left(\left(1-\mu_{A}\left(x_{i}\right)\right)^{\alpha}-\left(1-\mu_{A}\left(x_{i}\right)\right)^{\alpha}\right)}\right.\right. \\
& \left.-\mu_{A}\left(x_{i}\right) e^{\left(\mu_{A}^{\alpha}\left(x_{i}\right)-\mu_{A}^{\alpha}\left(x_{i}\right)\right)}\right] \\
& +\sum_{X_{2}}\left[1-\left(1-\mu_{B}\left(x_{i}\right)\right) e^{\left(\left(1-\mu_{A}\left(x_{i}\right)\right)^{\alpha}-\left(1-\mu_{B}\left(x_{i}\right)\right)^{\alpha}\right)}\right. \\
& \left.\left.-\mu_{B}\left(x_{i}\right) e^{\left(\mu_{A}^{\alpha}\left(x_{i}\right)-\mu_{B}^{\alpha}\left(x_{i}\right)\right)}\right]\right\} \\
& +\left\{\sum _ { X _ { 1 } } \left[1-\left(1-\mu_{B}\left(x_{i}\right)\right) e^{\left(\left(1-\mu_{A}\left(x_{i}\right)\right)^{\alpha}-\left(1-\mu_{B}\left(x_{i}\right)\right)^{\alpha}\right)}\right.\right. \\
& \left.-\mu_{B}\left(x_{i}\right) e^{\left(\mu_{A}^{\alpha}\left(x_{i}\right)-\mu_{B}^{\alpha}\left(x_{i}\right)\right)}\right] \\
& +\sum_{X_{2}}\left[1-\left(1-\mu_{A}\left(x_{i}\right)\right) e^{\left(\left(1-\mu_{A}\left(x_{i}\right)\right)^{\alpha}-\left(1-\mu_{A}\left(x_{i}\right)\right)^{\alpha}\right)}\right. \\
& \left.\left.-\mu_{A}\left(x_{i}\right) e^{\left(\mu_{A}^{\alpha}\left(x_{i}\right)-\mu_{A}^{\alpha}\left(x_{i}\right)\right)}\right]\right\} \\
& =\sum_{X_{1}}\left[1-\left(1-\mu_{B}\left(x_{i}\right)\right) e^{\left(\left(1-\mu_{A}\left(x_{i}\right)\right)^{\alpha}-\left(1-\mu_{B}\left(x_{i}\right)\right)^{\alpha}\right)}\right. \\
& \left.-\mu_{B}\left(x_{i}\right) e^{\left(\mu_{A}^{\alpha}\left(x_{i}\right)-\mu_{B}^{\alpha}\left(x_{i}\right)\right)}\right] \\
& +\sum_{X_{2}}\left[1-\left(1-\mu_{B}\left(x_{i}\right)\right) e^{\left(\left(1-\mu_{A}\left(x_{i}\right)\right)^{\alpha}-\left(1-\mu_{B}\left(x_{i}\right)\right)^{\alpha}\right)}\right. \\
& \left.-\mu_{B}\left(x_{i}\right) e^{\left(\mu_{A}^{\alpha}\left(x_{i}\right)-\mu_{B}^{\alpha}\left(x_{i}\right)\right)}\right] \\
& =\sum_{i=1}^{n}\left[1-\left(1-\mu_{B}\left(x_{i}\right)\right) e^{\left(\left(1-\mu_{A}\left(x_{i}\right)\right)^{\alpha}-\left(1-\mu_{B}\left(x_{i}\right)\right)^{\alpha}\right)}\right. \\
& \left.-\mu_{B}\left(x_{i}\right) e^{\left(\mu_{A}^{\alpha}\left(x_{i}\right)-\mu_{B}^{\alpha}\left(x_{i}\right)\right)}\right]=I_{E_{\alpha}}(B, A)
\end{aligned}
$$

Thus, $I_{E_{\alpha}}(A \cup B, A)+I_{E_{\alpha}}(A \cap B, A)=I_{E_{\alpha}}(B, A)$

Hence, the proof of 2 (a) holds. 


$$
\begin{aligned}
& 2(\mathrm{~b}) I_{E_{\alpha}}(A \cup B, C)+I_{E_{\alpha}}(A \cap B, C) \\
&=\sum_{i=1}^{n}\left[1-\left(1-\mu_{A \cup B}\left(x_{i}\right)\right) e^{\left(\left(1-\mu_{C}\left(x_{i}\right)\right)^{\alpha}-\left(1-\mu_{A \cup B}\left(x_{i}\right)\right)^{\alpha}\right)}-\mu_{A \cup B}\left(x_{i}\right) e^{\left(\mu_{C}^{\alpha}\left(x_{i}\right)-\mu_{A \cup B}^{\alpha}\left(x_{i}\right)\right)}\right] \\
& \quad+\sum_{i=1}^{n}\left[1-\left(1-\mu_{A \cap B}\left(x_{i}\right)\right) e^{\left(\left(1-\mu_{C}\left(x_{i}\right)\right)^{\alpha}-\left(1-\mu_{A \cap B}\left(x_{i}\right)\right)^{\alpha}\right)}-\mu_{A \cap B}\left(x_{i}\right) e^{\left(\mu_{C}^{\alpha}\left(x_{i}\right)-\mu_{A \cap B}^{\alpha}\left(x_{i}\right)\right)}\right] \\
&=\sum_{X_{1}}\left[1-\left(1-\mu_{A}\left(x_{i}\right)\right) e^{\left(\left(1-\mu_{C}\left(x_{i}\right)\right)^{\alpha}-\left(1-\mu_{A}\left(x_{i}\right)\right)^{\alpha}\right)}-\mu_{A}\left(x_{i}\right) e^{\left(\mu_{C}^{\alpha}\left(x_{i}\right)-\mu_{A}^{\alpha}\left(x_{i}\right)\right)}\right] \\
& \quad+\sum_{X_{2}}\left[1-\left(1-\mu_{B}\left(x_{i}\right)\right) e^{\left(\left(1-\mu_{C}\left(x_{i}\right)\right)^{\alpha}-\left(1-\mu_{B}\left(x_{i}\right)\right)^{\alpha}\right)}-\mu_{B}\left(x_{i}\right) e^{\left(\mu_{C}^{\alpha}\left(x_{i}\right)-\mu_{B}^{\alpha}\left(x_{i}\right)\right)}\right] \\
& \quad+\sum_{X_{1}}\left[1-\left(1-\mu_{B}\left(x_{i}\right)\right) e^{\left(\left(1-\mu_{C}\left(x_{i}\right)\right)^{\alpha}-\left(1-\mu_{B}\left(x_{i}\right)\right)^{\alpha}\right)}-\mu_{B}\left(x_{i}\right) e^{\left(\mu_{C}^{\alpha}\left(x_{i}\right)-\mu_{B}^{\alpha}\left(x_{i}\right)\right)}\right] \\
& \quad+\sum_{X_{2}}\left[1-\left(1-\mu_{A}\left(x_{i}\right)\right) e^{\left(\left(1-\mu_{C}\left(x_{i}\right)\right)^{\alpha}-\left(1-\mu_{A}\left(x_{i}\right)\right)^{\alpha}\right)}-\mu_{A}\left(x_{i}\right) e^{\left(\mu_{C}^{\alpha}\left(x_{i}\right)-\mu_{A}^{\alpha}\left(x_{i}\right)\right)}\right] \\
&= \sum_{i=1}^{n}\left[1-\left(1-\mu_{A}\left(x_{i}\right)\right) e^{\left(\left(1-\mu_{C}\left(x_{i}\right)\right)^{\alpha}-\left(1-\mu_{A}\left(x_{i}\right)\right)^{\alpha}\right)}-\mu_{A}\left(x_{i}\right) e^{\left(\mu_{C}^{\alpha}\left(x_{i}\right)-\mu_{A}^{\alpha}\left(x_{i}\right)\right)}\right] \\
&+\sum_{i=1}^{n}\left[1-\left(1-\mu_{B}\left(x_{i}\right)\right) e^{\left(\left(1-\mu_{C}\left(x_{i}\right)\right)^{\alpha}-\left(1-\mu_{B}\left(x_{i}\right)\right)^{\alpha}\right)}-\mu_{B}\left(x_{i}\right) e^{\left(\mu_{C}^{\alpha}\left(x_{i}\right)-\mu_{B}^{\alpha}\left(x_{i}\right)\right)}\right] \\
&= I_{E_{\alpha}}(A, C)+I_{E_{\alpha}}(B, C)
\end{aligned}
$$

Hence, the proof of 2 (b) holds.

$$
\begin{aligned}
& 2(\mathrm{c}) I_{E_{\alpha}}(\overline{A \cup B}, \overline{A \cap B})=\sum_{i=1}^{n}\left[1-\left(1-\mu_{\overline{A \cup B}}\left(x_{i}\right)\right) e^{\left(\left(1-\mu_{\overline{A \cap B}}\left(x_{i}\right)\right)^{\alpha}-\left(1-\mu_{\overline{A \cup B}}\left(x_{i}\right)\right)^{\alpha}\right)}\right. \\
& \left.-\mu_{\overline{A \cup B}}\left(x_{i}\right) e^{\left(\mu_{A \cap B}^{\alpha}\left(x_{i}\right)-\mu_{A \cup B}^{\alpha}\left(x_{i}\right)\right)}\right] \\
& =\sum_{i=1}^{n}\left[1-\mu_{A \cup B}\left(x_{i}\right) e^{\left(\mu_{A \cap B}^{\alpha}\left(x_{i}\right)-\mu_{A \cup B}^{\alpha}\left(x_{i}\right)\right)}-\left(1-\mu_{A \cup B}\left(x_{i}\right)\right) e^{\left(\left(1-\mu_{A \cap B}\left(x_{i}\right)\right)^{\alpha}-\left(1-\mu_{A \cup B}\left(x_{i}\right)\right)^{\alpha}\right)}\right] \\
& =\sum_{X_{1}}\left[1-\mu_{A}\left(x_{i}\right) e^{\left(\mu_{B}^{\alpha}\left(x_{i}\right)-\mu_{A}^{\alpha}\left(x_{i}\right)\right)}-\left(1-\mu_{A}\left(x_{i}\right)\right) e^{\left(\left(1-\mu_{B}\left(x_{i}\right)\right)^{\alpha}-\left(1-\mu_{A}\left(x_{i}\right)\right)^{\alpha}\right)}\right] \\
& +\sum_{X_{2}}\left[1-\mu_{B}\left(x_{i}\right) e^{\left(\mu_{A}^{\alpha}\left(x_{i}\right)-\mu_{B}^{\alpha}\left(x_{i}\right)\right)}-\left(1-\mu_{B}\left(x_{i}\right)\right) e^{\left(\left(1-\mu_{A}\left(x_{i}\right)\right)^{\alpha}-\left(1-\mu_{B}\left(x_{i}\right)\right)^{\alpha}\right)}\right]
\end{aligned}
$$

and

$$
\begin{aligned}
I_{E_{\alpha}}(\bar{A} \cap \bar{B}, \bar{A} \cup \bar{B})= & \sum_{i=1}^{n}\left[1-\left(1-\mu_{\bar{A} \cap \bar{B}}\left(x_{i}\right)\right) e^{\left(\left(1-\mu_{\bar{A} \cup \bar{B}}\left(x_{i}\right)\right)^{\alpha}-\left(1-\mu_{\bar{A} \cap \bar{B}}\left(x_{i}\right)\right)^{\alpha}\right)}-\mu_{\bar{A} \cap \bar{B}}\left(x_{i}\right) e^{\left(\mu_{\bar{A} \cup \bar{B}}^{\alpha}\left(x_{i}\right)-\mu_{\bar{A} \cap \bar{B}}^{\alpha}\left(x_{i}\right)\right)}\right] \\
= & \sum_{X_{1}}\left[1-\left(1-\mu_{\bar{A}}\left(x_{i}\right)\right) e^{\left(\left(1-\mu \bar{B}\left(x_{i}\right)\right)^{\alpha}-\left(1-\mu \bar{A}\left(x_{i}\right)\right)^{\alpha}\right)}-\mu_{\bar{A}}\left(x_{i}\right) e^{\left(\mu_{\bar{B}}^{\alpha}\left(x_{i}\right)-\mu \bar{A}^{\alpha}\left(x_{i}\right)\right)}\right] \\
& +\sum_{X_{2}}\left[1-\left(1-\mu_{\bar{B}}\left(x_{i}\right)\right) e^{\left(\left(1-\mu \bar{A}\left(x_{i}\right)\right)^{\alpha}-\left(1-\mu \bar{B}\left(x_{i}\right)\right)^{\alpha}\right)}-\mu_{\bar{B}}\left(x_{i}\right) e^{\left(\mu_{\bar{A}}^{\alpha}\left(x_{i}\right)-\mu \bar{B}^{\alpha}\left(x_{i}\right)\right)}\right] \\
= & \sum_{X_{1}}\left[1-\mu_{A}\left(x_{i}\right) e^{\left(\mu_{B}^{\alpha}\left(x_{i}\right)-\mu_{A}^{\alpha}\left(x_{i}\right)\right)}-\left(1-\mu_{A}\left(x_{i}\right)\right) e^{\left(\left(1-\mu_{B}\left(x_{i}\right)\right)^{\alpha}-\left(1-\mu_{A}\left(x_{i}\right)\right)^{\alpha}\right)}\right] \\
& +\sum_{X_{2}}\left[1-\mu_{B}\left(x_{i}\right) e^{\left(\mu_{A}^{\alpha}\left(x_{i}\right)-\mu_{B}^{\alpha}\left(x_{i}\right)\right)}-\left(1-\mu_{B}\left(x_{i}\right)\right) e^{\left(\left(1-\mu_{A}\left(x_{i}\right)\right)^{\alpha}-\left(1-\mu_{B}\left(x_{i}\right)\right)^{\alpha}\right)}\right] \\
= & I_{E_{\alpha}}(\overline{A \cup B}, \overline{A \cap B})
\end{aligned}
$$

Thus, $I_{E_{\alpha}}(\overline{A \cup B}, \overline{A \cap B})=I_{E_{\alpha}}(\bar{A} \cap \bar{B}, \bar{A} \cup \bar{B})$

Hence, the proof of $2(\mathrm{c})$ holds.

Theorem 3 (a) $I_{E_{\alpha}}(A, \bar{A})=I_{E_{\alpha}}(\bar{A}, A)$

(b) $I_{E_{\alpha}}(\bar{A}, \bar{B})=I_{E_{\alpha}}(A, B)$

(c) $I_{E_{\alpha}}(A, \bar{B})=I_{E_{\alpha}}(\bar{A}, B)$

(d) $I_{E_{\alpha}}(A, B)+I_{E_{\alpha}}(\bar{A}, B)=I_{E_{\alpha}}(\bar{A}, \bar{B})+I_{E_{\alpha}}(A, \bar{B})$ 


$$
\begin{aligned}
\operatorname{Proof} 3(\mathrm{a}) I_{E_{\alpha}}(A, \bar{A}) & =\sum_{i=1}^{n}\left[1-\left(1-\mu_{A}\left(x_{i}\right)\right) e^{\left(\left(1-\mu \bar{A}\left(x_{i}\right)\right)^{\alpha}-\left(1-\mu_{A}\left(x_{i}\right)\right)^{\alpha}\right)}-\mu_{A}\left(x_{i}\right) e^{\left(\mu \bar{A}^{\alpha}\left(x_{i}\right)-\mu_{A}^{\alpha}\left(x_{i}\right)\right)}\right] \\
& \left.=\sum_{i=1}^{n}\left[1-\left(1-\mu_{A}\left(x_{i}\right)\right) e^{\mu_{A}^{\alpha}\left(x_{i}\right)-\left(1-\mu_{A}\left(x_{i}\right)\right)^{\alpha}}\right)-\mu_{A}\left(x_{i}\right) e^{\left(\left(1-\mu_{A}\left(x_{i}\right)\right)^{\alpha}-\mu_{A}^{\alpha}\left(x_{i}\right)\right)}\right]
\end{aligned}
$$

and

$$
\begin{aligned}
I_{E_{\alpha}}(\bar{A}, A) & =\sum_{i=1}^{n}\left[1-\left(1-\mu_{\bar{A}}\left(x_{i}\right)\right) e^{\left(\left(1-\mu_{A}\left(x_{i}\right)\right)^{\alpha}-\left(1-\mu \bar{A}\left(x_{i}\right)\right)^{\alpha}\right)}-\mu_{\bar{A}}\left(x_{i}\right) e^{\left(\mu_{A}^{\alpha}\left(x_{i}\right)-\mu \bar{A}^{\alpha}\left(x_{i}\right)\right)}\right] \\
& =\sum_{i=1}^{n}\left[1-\mu_{A}\left(x_{i}\right) e^{\left(\left(1-\mu_{A}\left(x_{i}\right)\right)^{\alpha}-\mu_{A}^{\alpha}\left(x_{i}\right)\right)}-\left(1-\mu_{A}\left(x_{i}\right)\right) e^{\left.\mu_{A}^{\alpha}\left(x_{i}\right)-\left(1-\mu_{A}\left(x_{i}\right)\right)^{\alpha}\right)}\right] \\
& =I_{E_{\alpha}}(A, \bar{A})
\end{aligned}
$$

Thus, $I_{E_{\alpha}}(A, \bar{A})=I_{E_{\alpha}}(\bar{A}, A)$

Hence, the proof of 3(a) holds.

$$
\begin{aligned}
3(\mathrm{~b}) I_{E_{\alpha}}(\bar{A}, \bar{B}) & =\sum_{i=1}^{n}\left[1-\left(1-\mu_{\bar{A}}\left(x_{i}\right)\right) e^{\left(\left(1-\mu \bar{B}\left(x_{i}\right)\right)^{\alpha}-\left(1-\mu \bar{A}\left(x_{i}\right)\right)^{\alpha}\right)}-\mu_{\bar{A}}\left(x_{i}\right) e^{\left(\mu \bar{B}^{\alpha}\left(x_{i}\right)-\mu \bar{A}^{\alpha}\left(x_{i}\right)\right)}\right] \\
& =\sum_{i=1}^{n}\left[1-\mu_{A}\left(x_{i}\right) e^{\left(\mu_{B}^{\alpha}\left(x_{i}\right)-\mu_{A}^{\alpha}\left(x_{i}\right)\right)}-\left(1-\mu_{A}\left(x_{i}\right)\right) e^{\left(\left(1-\mu_{B}\left(x_{i}\right)\right)^{\alpha}-\left(1-\mu_{A}\left(x_{i}\right)\right)^{\alpha}\right)}\right] \\
& =I_{E_{\alpha}}(A, B)
\end{aligned}
$$

Thus, $I_{E_{\alpha}}(\bar{A}, \bar{B})=I_{E_{\alpha}}(A, B)$

Hence, the proof of 3(b) holds.

$$
\begin{aligned}
3(\mathrm{c}) I_{E_{\alpha}}(A, \bar{B}) & =\sum_{i=1}^{n}\left[1-\left(1-\mu_{A}\left(x_{i}\right)\right) e^{\left(\left(1-\mu \bar{B}\left(x_{i}\right)\right)^{\alpha}-\left(1-\mu_{A}\left(x_{i}\right)\right)^{\alpha}\right)}-\mu_{A}\left(x_{i}\right) e^{\left(\mu \bar{B}^{\alpha}\left(x_{i}\right)-\mu_{A}^{\alpha}\left(x_{i}\right)\right)}\right] \\
= & \left.\sum_{i=1}^{n}\left[1-\left(1-\mu_{A}\left(x_{i}\right)\right) e^{\mu_{B}^{\alpha}\left(x_{i}\right)-\left(1-\mu_{A}\left(x_{i}\right)\right)^{\alpha}}\right)-\mu_{A}\left(x_{i}\right) e^{\left(\left(1-\mu_{B}\left(x_{i}\right)\right)^{\alpha}-\mu_{A}^{\alpha}\left(x_{i}\right)\right)}\right] \\
\text { Now, } I_{E_{\alpha}}(\bar{A}, B) & =\sum_{i=1}^{n}\left[1-\left(1-\mu_{\bar{A}}\left(x_{i}\right)\right) e^{\left(\left(1-\mu_{B}\left(x_{i}\right)\right)^{\alpha}-\left(1-\mu \bar{A}\left(x_{i}\right)\right)^{\alpha}\right)}-\mu_{\bar{A}}\left(x_{i}\right) e^{\left(\mu_{B}^{\alpha}\left(x_{i}\right)-\mu \bar{A}^{\alpha}\left(x_{i}\right)\right)}\right] \\
& =\sum_{i=1}^{n}\left[1-\mu_{A}\left(x_{i}\right) e^{\left(\left(1-\mu_{B}\left(x_{i}\right)\right)^{\alpha}-\mu_{A}^{\alpha}\left(x_{i}\right)\right)}-\left(1-\mu_{A}\left(x_{i}\right)\right) e^{\left.\mu_{B}^{\alpha}\left(x_{i}\right)-\left(1-\mu_{A}\left(x_{i}\right)\right)^{\alpha}\right)}\right] \\
& =I_{E_{\alpha}}(A, \bar{B})
\end{aligned}
$$

Thus, $I_{E_{\alpha}}(A, \bar{B})=I_{E_{\alpha}}(\bar{A}, B)$

Hence, the proof of 3(c) holds.

3(d) It obviously follows from 3(b) and 3(c).

Theorem 4 Relation between $E_{\alpha}(A)$ and $I_{E_{\alpha}}(A, B)$ is given by

$$
E_{\alpha}(A)=1-\frac{e^{1-0.5^{\alpha}}}{n\left(e^{1-0.5^{\alpha}}-1\right)} I_{E_{\alpha}}\left(A,\left[\frac{1}{2}\right]\right)
$$




$$
\begin{aligned}
\operatorname{Proof} I_{E_{\alpha}}\left(A,\left[\frac{1}{2}\right]\right)=\sum_{i=1}^{n}\left[1-\left(1-\mu_{A}\left(x_{i}\right)\right) e^{\left(0.5^{\alpha}-\left(1-\mu_{A}\left(x_{i}\right)\right)^{\alpha}\right)}-\mu_{A}\left(x_{i}\right) e^{\left(0.5^{\alpha}-\mu_{A}^{\alpha}\left(x_{i}\right)\right)}\right] \\
=\sum_{i=1}^{n}\left[1-\left(1-\mu_{A}\left(x_{i}\right)\right) \frac{e^{-\left(1-\mu_{A}\left(x_{i}\right)\right)^{\alpha}}}{e^{-0.5^{\alpha}}}-\mu_{A}\left(x_{i}\right) \frac{e^{-\mu_{A}^{\alpha}\left(x_{i}\right)}}{e^{-0.5^{\alpha}}}\right] \\
=\sum_{i=1}^{n}\left[1-\left(1-\mu_{A}\left(x_{i}\right)\right) \frac{e^{1-\left(1-\mu_{A}\left(x_{i}\right)\right)^{\alpha}}}{e^{1-0.5^{\alpha}}}-\mu_{A}\left(x_{i}\right) \frac{e^{1-\mu_{A}^{\alpha}\left(x_{i}\right)}}{e^{1-0.5^{\alpha}}}\right] \\
=n-\frac{1}{e^{\left(1-0.5^{\alpha}\right)}} \sum_{i=1}^{n}\left[\left(1-\mu_{A}\left(x_{i}\right)\right) e^{\left(1-\left(1-\mu_{A}\left(x_{i}\right)\right)^{\alpha}\right)}+\mu_{A}\left(x_{i}\right) e^{\left(1-\mu_{A}^{\alpha}\left(x_{i}\right)\right)}+1-1\right] \\
=n-\frac{1}{e^{\left(1-0.5^{\alpha}\right)}} \sum_{i=1}^{n}\left[\mu_{A}\left(x_{i}\right) e^{\left(1-\mu_{A}^{\alpha}\left(x_{i}\right)\right)}+\left(1-\mu_{A}\left(x_{i}\right)\right) e^{1-\left(1-\mu_{A}\left(x_{i}\right)\right)^{\alpha}}-1\right]-\frac{n}{e^{\left(1-0.5^{\alpha}\right)}} \\
=n-\frac{1}{e^{\left(1-0.5^{\alpha}\right)}} n\left(e^{\left(1-0.5^{\alpha}\right)}-1\right) E_{\alpha}(A)-\frac{n}{e^{\left(1-0.5^{\alpha}\right)}} \\
=n\left(1-\frac{1}{e^{\left(1-0.5^{\alpha}\right)}}\right)-\frac{1}{e^{\left(1-0.5^{\alpha}\right)}} n\left(e^{\left(1-0.5^{\alpha}\right)}-1\right) E_{\alpha}(A) \\
=\frac{n\left(e^{\left(1-0.5^{\alpha}\right)}-1\right)}{e^{\left(1-0.5^{\alpha}\right)}}-\frac{n\left(e^{\left(1-0.5^{\alpha}\right)}-1\right)}{e^{\left(1-0.5^{\alpha}\right)}} E_{\alpha}(A)=\left(1-E_{\alpha}(A)\right) \frac{n\left(e^{\left(1-0.5^{\alpha}\right)}-1\right)}{e^{\left(1-0.5^{\alpha}\right)}} \\
\Rightarrow \frac{e^{\left(1-0.5^{\alpha}\right)}}{n\left(e^{\left(1-0.5^{\alpha}\right)}-1\right)} I_{E_{\alpha}}\left(A,\left[\frac{1}{2}\right]\right)=\left(1-E_{\alpha}(A)\right) \\
\operatorname{Thus,} E_{\alpha(A)}=1-\frac{e^{\left(1-0.5^{\alpha}\right)}}{n\left(e^{\left(1-0.5^{\alpha}\right)}-1\right)} I_{E_{\alpha}}\left(A,\left[\frac{1}{2}\right]\right)
\end{aligned}
$$

Hence, the proof of theorem 4 holds.

\section{A comparative study}

In this section, we demonstrate the efficiency of proposed fuzzy divergence measure by comparing it with some of existing fuzzy divergence measures. To do so, we present the comparative study of the proposed divergence measure with the existing fuzzy divergence measures given by Kapur [3], Prakash et al. [12], and Bajaj and Hooda [16].

Let $\mathrm{A}$ and $\mathrm{B}$ be any two fuzzy sets given $\mathrm{A}=\{0.1,0.9,0.5\}, \mathrm{B}=\{0.6,0.7,0.1\}$.

The computed values of fuzzy divergence measures $\bar{I}_{\alpha}(A: B), I_{a}(A: B), D_{\alpha}(A, B), I_{E_{\alpha}}$ $(A, B)$ are presented in Table 1.

Table 1 and Figure 1 depict the minimization of degree of difference of the proposed fuzzy measure. It is clear that the proposed fuzzy divergence measure is efficient than the existing fuzzy divergence measures.

\section{Application of parametric generalized exponential measure of fuzzy divergence in strategic decision making}

As we have already discussed in the introductory section, in recent years, the applications of the fuzzy divergence measure have been given in different areas: Poletti et al. [23] in bio-informatics; Bhandari et al. [21], Fan et al. [24], and Bhatia and Singh [25] in image thresholding; and Ghosh et al. [10] in automated leukocyte recognition. We

Table 1 Computed values of fuzzy divergence measures: $I_{a}(A, B), D_{a}(A, B), I_{a}(A: B), I_{E_{a}}(A, B)$

\begin{tabular}{lccccc}
\hline Fuzzy divergence measure & $\boldsymbol{a}, \boldsymbol{a}=\mathbf{0 . 1}$ & $\boldsymbol{a}, \boldsymbol{a}=\mathbf{0 . 4}$ & $\boldsymbol{a}, \boldsymbol{a}=\mathbf{0 . 5}$ & $\boldsymbol{a}, \boldsymbol{a}=\mathbf{0 . 6}$ & $\boldsymbol{a}, \boldsymbol{a}=\mathbf{0 . 9}$ \\
\hline$I_{a}(A, B)$ & 0.1243 & 0.4743 & 0.5875 & 0.7006 & 1.4809 \\
$D_{a}(A, B)$ & 0.1276 & 0.5058 & 0.6273 & 0.7456 & 1.0766 \\
$I_{a}(A: B)$ & 1.1734 & 1.0272 & 0.9966 & 0.9681 & 0.8933 \\
$I_{E_{\alpha}}(A, B)$ & 0.0977 & 0.2406 & 0.2805 & 0.3017 & 0.3416 \\
\hline
\end{tabular}




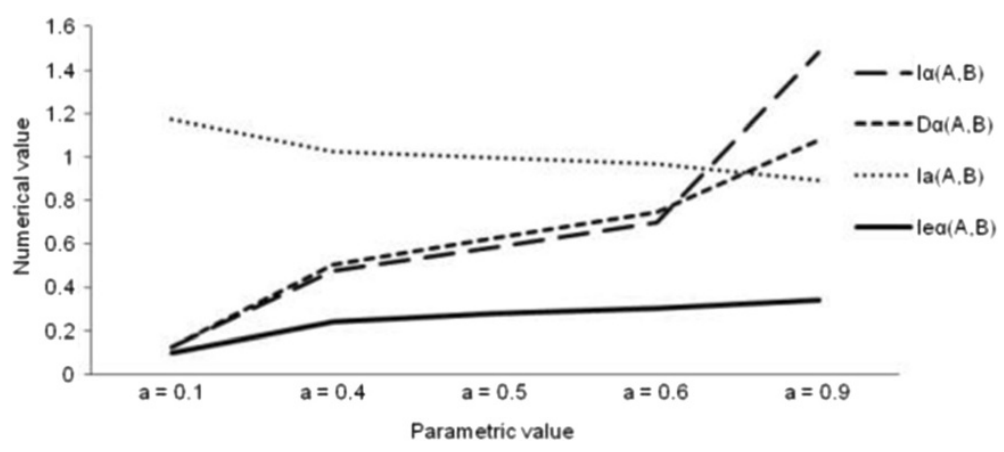

Figure 1 Comparison of numerical values of fuzzy divergence measures $I_{a}(A, B), D_{a}(A, B), I_{a}(A: B)$, $\mathbf{I}_{\mathbf{E}_{\alpha}}(\mathbf{A}, \mathbf{B})$.

provide an application of the proposed divergence measure in strategic decisionmaking.

Decision-making problem is the process of finding the best option from all of the feasible alternatives. It is assumed that a firm $X$ desire to apply $m$ strategies $S_{1}, S_{2}, S_{3}, \ldots, S_{m}$ to meet its goal. Let each strategy have different degree of effectiveness if the input associated with it is varied and let $\left\{I_{1}, I_{2}, I_{3}, \ldots, I_{n}\right\}$ be the input set or set of alternatives. Let the fuzzy set $Y$ denotes the effectiveness of a particular strategy with uniform input. Therefore,

$$
Y=\left\{\left(Y, \mu_{Y}\left(S_{i}\right)\right) / i=1,2, \ldots, m\right\}
$$

Further, let $I_{j}$ be a fuzzy set denotes the degree of effectiveness of a strategy when it is implemented with input $I_{j}$.

$$
I_{j}=\left\{\left(I_{j}, \mu_{I_{j}}\left(S_{i}\right)\right) / i=1,2, \ldots, m\right\} \text { where } j=1,2, \ldots, n \text {. }
$$

Taking $A=Y$ and $B=I_{j}$ in the fuzzy divergence measure $I_{E_{\alpha}}\left(Y, I_{j}\right)$ defined in new parametric generalized exponential measure of fuzzy divergence section, and we calculate $I_{E_{\alpha}}\left(Y, I_{j}\right)$.

Then, most effective $I_{j}$ is determined by $\operatorname{Min}\left\{I_{E_{\alpha}}\left(Y, I_{j}\right)\right\}_{\substack{1 \leq j \leq n \\ 0<\alpha \leq 0.5}}$. It is assumed that $I_{t}(1 \leq t \leq n)$ determines the minimum value of $\left\{I_{E_{\alpha}}\left(Y, I_{j}\right)\right\}_{0<\alpha \leq 0.5}$. With this $I_{t}$ find $\operatorname{Max} .\left\{\mu_{I_{j}}\left(S_{i}\right)\right\}_{1 \leq i \leq m}$, let it correspond to $S_{p}, 1 \leq p \leq m$.

Hence, if the strategy $S_{p}$ is implemented with input budget of $I_{t}$, the firm will meet its goal in the most input-effective manner.

\section{An illustrative example}

Let $m=n=5$ in the above model. Table 2 shows the efficiency of different strategies at uniform inputs.

Table 3 illustrates the efficiency of different strategies at particular inputs.

Table 2 Efficiency of different strategies at uniform inputs

\begin{tabular}{lcccc}
\hline$\mu_{Y}\left(S_{1}\right)$ & $\mu_{Y}\left(S_{2}\right)$ & $\mu_{Y}\left(S_{3}\right)$ & $\mu_{Y}\left(S_{4}\right)$ & $\mu_{Y}\left(S_{5}\right)$ \\
\hline 0.5 & 0.3 & 0.6 & 0.4 & 0.7 \\
\hline
\end{tabular}


Table 3 Efficiency of strategies at particular inputs

\begin{tabular}{cccccc}
\hline $\boldsymbol{I}_{\boldsymbol{j}}$ & $\boldsymbol{\mu}_{\mathbf{l}_{\mathbf{j}}}\left(\boldsymbol{S}_{\mathbf{1}}\right)$ & $\boldsymbol{\mu}_{\boldsymbol{l}_{j}}\left(\boldsymbol{S}_{\mathbf{2}}\right)$ & $\boldsymbol{\mu}_{\mathbf{l}_{\mathrm{j}}}\left(\boldsymbol{S}_{\mathbf{3}}\right)$ & $\boldsymbol{\mu}_{\boldsymbol{l}_{\mathbf{j}}}\left(\boldsymbol{S}_{\mathbf{4}}\right)$ & $\boldsymbol{\mu}_{\boldsymbol{l}_{\mathbf{j}}}\left(\boldsymbol{S}_{\mathbf{5}}\right)$ \\
\hline$I_{1}$ & 0.3 & 0.4 & 0.5 & 0.8 & 0.6 \\
$I_{2}$ & 0.6 & 0.3 & 0.9 & 0.2 & 0.4 \\
$I_{3}$ & 0.7 & 0.5 & 0.8 & 0.9 & 0.6 \\
$I_{4}$ & 0.8 & 0.7 & 0.6 & 0.5 & 0.3 \\
$I_{5}$ & 0.5 & 0.8 & 0.4 & 0.7 & 0.5 \\
\hline
\end{tabular}

The numerical values of divergence measure $\left.I_{E_{\alpha}}\left(Y, I_{j}\right)\right\}_{\substack{1 \leq j \leq n \\ 0<\alpha \leq 0.5}}$ are presented in Table 4.

The calculated numerical values of the proposed fuzzy divergence measure indicate that alternative input $I_{2}$ is more appropriate for different values of $\alpha(0<\alpha \leq 0.5)$. An examination of the results presented in Tables 3 and 4 makes it clear that strategy $S_{4}$ is most effective. Thus, a firm will achieve its goal most effectively if the strategy $S_{4}$ is applied with an input alternative $I_{2}$.

\section{Application of fuzzy TOPSIS and MOORA methods for strategic decision making: a} comparative analysis $^{a}$

We now present the application of TOPSIS [26] and MOORA [27] methods for strategic decision-making using the proposed fuzzy divergence measure (6).

\section{Fuzzy TOPSIS method}

Let us assume that there exists a set $I=\left\{I_{1}, I_{2}, I_{3}, \ldots, I_{n}\right\}$ of $n$ alternative inputs and a set of $m$ attributes (strategies) given by $S=\left\{S_{1}, S_{2}, S_{3}, \ldots, S_{m}\right\}$. The decision maker has to find the best alternative from the set $I$ corresponding to set $S$ of $n$ attributes (strategies).

The computational procedure of solving the fuzzy strategic decision-making problem involves the following steps:

1. Construct a fuzzy decision matrix.

$$
\begin{array}{lcccc} 
& S_{1} & S_{2} & \ldots & S_{n} \\
I_{1} & x_{11} & x_{12} & \ldots & x_{1 n} \\
I_{2} & x_{21} & x_{22} & \ldots & x_{2 n} \\
I_{m} & x_{m 1} & x_{m 2} & \ldots & x_{m n} \\
W=\left[w_{1}, w_{2}, \ldots, w_{n}\right]
\end{array}
$$

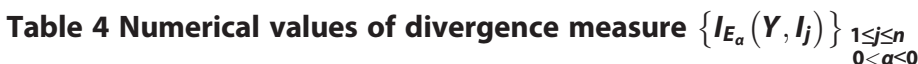

\begin{tabular}{lcccc}
\hline $\boldsymbol{I}_{\boldsymbol{j}}$ & $\boldsymbol{a}=\mathbf{0 . 1}$ & $\boldsymbol{a}=\mathbf{0 . 3}$ & $\boldsymbol{a}=\mathbf{0 . 4}$ & $\boldsymbol{a}=\mathbf{0 . 5}$ \\
\hline$I_{1}$ & 0.0456 & 0.0831 & 0.0900 & 0.0916 \\
$I_{2}$ & 0.0449 & 0.0662 & 0.0568 & 0.0396 \\
$I_{3}$ & 0.0779 & 0.1308 & 0.1289 & 0.1164 \\
$I_{4}$ & 0.0748 & 0.1500 & 0.1646 & 0.1700 \\
$I_{5}$ & 0.0764 & 0.1568 & 0.1748 & 0.1843 \\
\hline
\end{tabular}


Table 5 Normalized/weighted normalized fuzzy decision-matrix

\begin{tabular}{llcccc}
\hline & $\boldsymbol{\mu}_{l_{j}}\left(\boldsymbol{S}_{\mathbf{1}}\right)$ & $\boldsymbol{\mu}_{\boldsymbol{\mu l}_{j}}\left(\boldsymbol{S}_{2}\right)$ & $\boldsymbol{\mu}_{\boldsymbol{l}_{j}}\left(\boldsymbol{S}_{\mathbf{3}}\right)$ & $\boldsymbol{\mu}_{\boldsymbol{l}_{j}}\left(\boldsymbol{S}_{\mathbf{4}}\right)$ & $\boldsymbol{\mu}_{\boldsymbol{l}_{j}}\left(\boldsymbol{S}_{\mathbf{5}}\right)$ \\
\hline$I_{1}$ & 0.2218 & 0.3133 & 0.3356 & 0.5010 & 0.5432 \\
$I_{2}$ & 0.4435 & 0.2350 & 0.6040 & 0.1252 & 0.3622 \\
$I_{3}$ & 0.5174 & 0.3916 & 0.5369 & 0.5636 & 0.5432 \\
$I_{4}$ & 0.5914 & 0.5483 & 0.4027 & 0.3131 & 0.2716 \\
$I_{5}$ & 0.3696 & 0.6266 & 0.2685 & 0.4383 & 0.4527 \\
\hline
\end{tabular}

2. Construct the normalized fuzzy decision matrix. The normalized value $n_{i j}$ is calculated as

$$
\text { as } n_{i j}=x_{i j} / \sqrt{\sum_{j=1}^{m} x_{i j}^{2}}, j=1, \ldots, m, i=1, \ldots, n
$$

3. Construct the weighted normalized fuzzy decision matrix, the weighted normalized value

$$
v_{i j}=w_{i} n_{i j}, j=1, \ldots, m, i=1, \ldots, n
$$

where weighted matrix for each strategy is as follows: $W=[1,1,1,1,1]$ and $w_{i}$ is the weight of $i$ th attribute.

4. Determine the fuzzy positive ideal and fuzzy negative ideal solution $A^{+}$and $A^{-}$, using the formulas

$$
\begin{aligned}
& \begin{aligned}
A^{+}=\{ & \left.v_{1}^{+}, v_{2}^{+}, \ldots, v_{n}^{+}\right\}=\left\{\max _{j} v_{i j} / i \in I\right\} \\
\text { and } A^{-} & =\left\{v_{1}^{-}, v_{2}^{-}, \ldots, v_{n}^{-}\right\} \\
& =\left\{\min _{j} v_{i j} / i \in I\right\} \text { respectively, where } I \text { is associated with input set. }
\end{aligned}
\end{aligned}
$$

5. Calculate the separation of each alternative input from positive ideal solution and negative ideal solution, respectively, using the proposed measure (6).

6. Calculate the relative closeness of each alternative to positive ideal solution using the formula

$$
R_{j}=I_{E_{\alpha}}^{-} /\left(I_{E_{\alpha}}^{+}+I_{E_{\alpha}}^{-}\right), j=1, \ldots, m
$$

7. Rank the preference order of all alternatives according to the closeness coefficient.

Now the application of proposed measure $I_{E_{\alpha}}(A, B)$ with TOPSIS technique is demonstrated using the fuzzy decision matrix considered in Table 3.

Table 5 presents the normalized/weighted normalized fuzzy decision matrix corresponding to the fuzzy decision matrix given in Table 3 using the formulas (9) and (10).

Table 6 Fuzzy positive and negative ideal solution

\begin{tabular}{lccccr}
\hline & $\mu_{l_{j}}\left(\boldsymbol{S}_{1}\right)$ & $\mu_{l_{j}}\left(\boldsymbol{S}_{2}\right)$ & $\mu_{l_{j}}\left(\boldsymbol{S}_{3}\right)$ & $\boldsymbol{\mu}_{l_{j}}\left(\boldsymbol{S}_{\mathbf{4}}\right)$ & $\boldsymbol{\mu}_{\boldsymbol{l}_{j}}\left(\boldsymbol{S}_{5}\right)$ \\
\hline$A^{+}$ & 0.5432 & 0.6040 & 0.5636 & 0.5914 & 0.6266 \\
$A^{-}$ & 0.2218 & 0.1252 & 0.3916 & 0.2716 & 0.2685 \\
\hline
\end{tabular}


Table 7 Distance of each alternative from positive and negative ideal solution

\begin{tabular}{|c|c|c|c|c|}
\hline & $I_{E_{a}}^{+}$ & & $I_{E_{\alpha}}^{-}$ & \\
\hline & $a=0.1$ & $a=0.5$ & $a=0.1$ & $a=0.5$ \\
\hline$I_{1}$ & 0.0463 & 0.1568 & 0.0296 & 0.0113 \\
\hline$I_{2}$ & 0.0819 & 0.2835 & 0.0263 & 0.0366 \\
\hline 13 & 0.0090 & 0.0173 & 0.0611 & 0.0511 \\
\hline$I_{4}$ & 0.0418 & 0.1338 & 0.0616 & 0.0732 \\
\hline$I_{5}$ & 0.0306 & 0.0986 & 0.0682 & 0.0847 \\
\hline
\end{tabular}

Table 6 shows the fuzzy positive and negative ideal solutions $A^{+}$and $A^{-}$using formulas (11) and (12).

The calculated numerical values of divergence/distance values of each alternative input from positive ideal solution and negative ideal solution using formula (6) are given in Table 7.

The best alternative is the one with the shortest distance to the fuzzy positive ideal solution and with the longest distance to the fuzzy negative ideal solution. The calculated values of relative closeness of each alternative to positive ideal solution using formula (13) and their corresponding ranks are shown in Table 8.

According to the closeness coefficient, the ranking of the preference order of these alternatives $I_{j}(j=1,2,3,4,5)$ :

$$
\begin{aligned}
& \text { For } \alpha=0.1, I_{3}>I_{5}>I_{4}>I_{1}>I_{2} . \\
& \text { For } \alpha=0.5, I_{3}>I_{5}>I_{4}>I_{2}>I_{1} .
\end{aligned}
$$

Thus, here, we find that variation in values of $\alpha$ brings about change in ranking, but leaves the best choice unchanged. So $I_{3}$ is the most preferable alternative.

\section{Fuzzy MOORA method}

Fuzzy MOORA method for solving strategic decision-making problems is as follows. The computational procedure in fuzzy MOORA method up to step 4 is the same as discussed in fuzzy TOPSIS method above.

Step 5. Calculate the overall performance index $I_{E_{\alpha}}\left(A^{+}, A^{-}\right)$for each alternative using the formula (6) and the computed values in Table 6.

Step 6. Ranking alternatives and/or selecting the most efficient one are based on the values of $I_{E_{\alpha}}\left(A^{+}, A^{-}\right)$.

The overall performance index $I_{E_{\alpha}}\left(A^{+}, A^{-}\right)$for each alternative is calculated using formula (6) and values given in Table 6. Finally, the ranking results have been obtained using MOORA method and are presented in Table 9.

Table 8 Closeness coefficient and ranking

\begin{tabular}{lcccc}
\hline Alternatives (inputs) & $\boldsymbol{a}=\mathbf{0 . 1}$ & Rank & $\boldsymbol{a}=\mathbf{0 . 5}$ & Rank \\
\hline$I_{1}$ & 0.3900 & 4 & 0.0672 & 5 \\
$I_{2}$ & 0.2431 & 5 & 0.1143 & 4 \\
$I_{3}$ & 0.8716 & 1 & 0.7471 & 1 \\
$I_{4}$ & 0.5957 & 3 & 0.3536 & 3 \\
$I_{5}$ & 0.6903 & 2 & 0.4621 & 2 \\
\hline
\end{tabular}


Table 9 The ranking results obtained using MOORA method

\begin{tabular}{lcccc}
\hline & & $\boldsymbol{I}_{\boldsymbol{E}_{\mathbf{a}}}\left(\mathbf{A}^{+}, \mathbf{A}^{-}\right)$ & Rank \\
\cline { 3 - 5 } & $\boldsymbol{a}=\mathbf{0 . 1}$ & $\mathbf{R a n k}$ & $\boldsymbol{a}=\mathbf{0 . 5}$ & 2 \\
$I_{1}$ & 0.9816 & 3 & 0.9778 & 5 \\
$I_{3}$ & 0.9528 & 5 & 0.9333 & 1 \\
$I_{4}$ & 0.9951 & 1 & 0.9891 & 3 \\
$I_{5}$ & 0.9823 & 2 & 0.9678 & 4 \\
\hline
\end{tabular}

According to the calculation results, ranking order of alternatives is as follows:

$$
\begin{aligned}
& \text { For } \alpha=0.1, I_{3}>I_{4}>I_{1}>I_{5}>I_{2} . \\
& \text { For } \alpha=0.5, I_{3}>I_{1}>I_{4}>I_{5}>I_{2} .
\end{aligned}
$$

Thus, here, we find that variation in values of $\alpha$ brings about change in ranking, but leaves the best choice unchanged. So $I_{3}$ is the most preferable alternative.

\section{A comparative analysis of the proposed method and the existing methods of strategic decision-making}

We now compare the proposed method of strategic decision-making with the existing method of strategic decision-making using the proposed measure (6). From the proposed method in the 'Application of parametric generalized exponential measure of fuzzy divergence in strategic decision making' section, it is clear that a firm will achieve its goal most effectively if the strategy $S_{4}$ is applied with an input alternative $I_{2}$. Thus, $I_{2}$ is best input alternative for a firm to achieve its goal. However, we above examine from the existing methods that $I_{3}$ is the most preferable input alternative. It is also noticed that the proposed method is a very short, simple, and consistent method than the existing methods of strategic decision-making having a computational procedure involving a number of steps. Thus, the proposed method of strategic decision-making is better than the existing methods.

\section{Concluding remarks}

In this paper, we have proposed and validated the generalized exponential measure of fuzzy divergence. We have established the relation between generalized exponential fuzzy entropy and the proposed fuzzy divergence measure. Particular case and some of the properties of this divergence measure are proved. The efficiency of the new divergence measure has been presented. In addition, application of the proposed divergence measure is discussed in strategic decision-making and a numerical example is given for illustration. The application of the new measure of fuzzy divergence in two existing methods of strategic decision-making is presented. A comparative analysis between the proposed method of strategic decision-making and the existing methods of strategic decision-making has also been provided. We note that our measure of fuzzy divergence comprises greater consistency and flexibility in applications because of the presence of the parameter. 


\section{Endnote}

${ }^{\text {a }}$ The authors are thankful to an anonymous referee of this journal for bringing this point to their attention.

\section{Authors' information}

Mathematics Subject Classification (2010) 94A17, 03E72, $90 \mathrm{~B} 50$.

\section{Acknowledgements}

The authors would like to thank the editor and two anonymous referees of this journal for their constructive suggestions. Thanks are also due to Dr. Ramphul Ohlan, Assistant Professor, Institute of Management Studies and Research, Maharshi Dayanand University, Rohtak 124 001, Haryana, India, for his comments to improve the readability of the paper.

Received: 18 August 2014 Accepted: 13 November 2014

Published online: 05 December 2014

\section{References}

1. Shannon, CE: A mathematical theory of communication. Bell. Syst. Tech. J. 27(3), 379-423 (1948). Accessed on September 19, 2014 http://cm.bell-labs.com/cm/ms/what/shannonday/shannon1948.pdf

2. Zadeh, LA: Fuzzy sets. Inf. Control. 8(3), 338-353 (1965)

3. Kapur, JN: Measures of Fuzzy Information. Mathematical Sciences Trust Society, New Delhi (1997).

4. De Luca, A, Termini, S: A definition of non-probabilistic entropy in the setting of fuzzy set theory. Inf. Control. 20(4), 301-312 (1972)

5. Bhandari, D, Pal, NR: Some new information measures for fuzzy sets. Inf. Sci. 67(3), 209-228 (1993)

6. Pal, NR, Pal, SK: Object background segmentation using new definition of entropy. IEEE Proc. 136(4), 284-295 (1989)

7. Verma, R, Sharma, BD: On generalized exponential fuzzy entropy. World Acad. Sci. Eng. Technol. 60, 1402-1405 (2011). Accessed on September 5, 2014 http://waset.org/publications/9417/on-generalized-exponential-fuzzyentropy

8. Kullback, S, Leibler, RA: On information and sufficiency. Ann. Math. Stat. 22(1), 79-86 (1951). Accessed on September 22, 2014 http://www.csee.wvu.edu/ xinl/library/papers/math/statistics/Kullback_Leibler_1951.pdf

9. Fan, J, Xie, W: Distance measures and induced fuzzy entropy. Fuzzy Sets Syst. 104(2), 305-314 (1999)

10. Ghosh, M, Das, D, Ray, C, Chakraborty, AK: Automated leukocyte recognition using fuzzy divergence. Micron 41, 840-846 (2010)

11. Montes, S, Couso, I, Gil, P, Bertoluzza, C: Divergence measure between fuzzy sets. Int. J. Approx. Reason. 30, 91-105 (2002)

12. Prakash, O, Sharma, PK, Kumar, S: Two new measures of fuzzy divergence and their properties. SQU J. Sci. 11, 69-77 (2006). Accessed on September 10, 2014 http://web.squ.edu.om/squjs/volum11/MATH041130-corrected.pdf

13. Ferreri, C: Hyper entropy and related heterogeneity divergence and information measures. Statistica 40(2), 155-168 (1980)

14. Renyi, A: On measures of entropy and information. Proc. 4th Berkeley Symp. Math. Stat. Probab. 1, 547-561 (1961)

15. Sharma, BD, Mittal, DP: New non-additive measures of entropy for discrete probability distributions. J. Math. Sci (Calcutta). 10, 28-40 (1975)

16. Bajaj, RK, Hooda, DS: On some new generalized measures of fuzzy information. World Acad. Sci. Eng. Technol. 62, 747-753 (2010)

17. Bhatia, PK, Singh, S: Three families of generalized fuzzy directed divergence. AMO 14(3), 599-614 (2012)

18. Taneja, IJ: On Mean Divergence Measures, pp. 1-18. (2005). Accessed on September 18, 2014 http://arxiv.org/pdf/ math/0501298v2.pdf

19. Zadeh, LA: Probability measures of fuzzy events. J. Math. Anal. Appl. 23, 421-427 (1968)

20. Bouchon-Meunier, B, Rifqi, M, Bothorel, S: Towards general measures of comparison of objects. Fuzzy Sets Syst. 84, 143-153 (1996)

21. Bhandari, D, Pal, NR, Majumder, DD: Fuzzy divergence, probability measure of fuzzy events and image thresholding. Inf. Sci. 13, 857-867 (1992)

22. Havrda, JH, Charvat, F: Quantification methods of classification processes: concepts of structural a entropy. Kybernetika 3, 30-35 (1967)

23. Poletti, E, Zappelli, F, Ruggeri, A, Grisan, E: A review of thresholding strategies applied to human chromosome segmentation. Comput. Methods Prog. Biomed. 108, 679-688 (2012)

24. Fan, S, Yang, S, He, P, Nie, H: Infrared electric image thresholding using two dimensional fuzzy entropy. Energey Procedia 12, 411-419 (2011)

25. Bhatia, PK, Singh, S: A new measure of fuzzy directed divergence and its application in image segmentation. Int. J. Intell. Sys. Appl. 4, 81-89 (2013). Accessed on September 12, 2014. http://www.mecs-press.org/ijisa/ijisa-v5-n4/ IJISA-V5-N4-8.pdf

26. Hwang, CL, Yoon, K: Multiple Attribute Decision Making: Methods and Applications. Springer, New York (1981)

27. Brauers, WKM, Zavadskas, EK: The MOORA method and its application to privatization in transition economy. Control. Cybern. 35(2), 443-468 (2006) 